\title{
Raman spectra of $(\mathrm{He})_{N}-\mathrm{Br}_{2}(X)$ clusters: The role of boson/fermion statistics in a quantum solvent
}

\author{
D. López-Durán, M. P. de Lara-Castells, G. Delgado-Barrio, and P. Villarreala) \\ Instituto de Matemáticas y Física Fundamental (C.S.I.C.), Serrano 123, E-28006-Madrid, Spain \\ C. Di. Paola and F. A. Gianturco \\ Department of Chemistry and INFM, The University of Rome, Città Universitaria, 00185 Rome, Italy \\ J. Jellinek \\ Chemistry Division, Argonne National Laboratory, Argonne, Illinois 60439
}

(Received 1 April 2004; accepted 17 May 2004)

\begin{abstract}
The aim of this paper is to elucidate the role played by the bosonic/fermionic character of $N \mathrm{He}$ atoms solvating a $\mathrm{Br}_{2}(X)$ molecule. To this end, an adiabatic model in the molecular stretching coordinate is assumed and the ground energy levels of the complexes are searched by means of Hartree (or Hartree-Fock) Quantum Chemistry calculations for ${ }^{4} \mathrm{He}$ (or ${ }^{3} \mathrm{He}$ ) solvent atoms. Simulations of vib-rotational Raman spectra point at the spin multiplicity as the main feature responsible for the drastic difference in the rotational structures of molecules embedded in boson or fermion helium drops as already observed by the experiments of Grebenev et al. [S. Grebenev, J. P. Toennies, and A. F. Vilesov, Science 279 (1998) 2083]. (C) 2004 American Institute of Physics.
\end{abstract}

[DOI: $10.1063 / 1.1769369$ ]

\section{INTRODUCTION}

The pioneering experiments of Grebenev et al. ${ }^{1}$ detected a tremendous difference in the infrared (IR) spectra of the oxygen carbon sulfide (OCS) molecule surrounded by helium atoms depending on the isotope considered, i.e., either ${ }^{4} \mathrm{He}$ or ${ }^{3} \mathrm{He}$. In that work, apart from the small shifts attributed to the weak OCS-He interactions and the broadening induced by rotational diffusion, the IR spectrum of OCS in pure ${ }^{4} \mathrm{He}$ droplets resembles the corresponding spectrum of isolated OCS with well-defined $P$ and $R$ branches. On the contrary, the spectrum obtained when ${ }^{3} \mathrm{He}$ droplets are used as solvent was found to be highly unstructured and showing a rather broad peak. Moreover, when mixtures of ${ }^{4} \mathrm{He} /{ }^{3} \mathrm{He}$ are used, the evolution of the spectra with the number of ${ }^{4} \mathrm{He}$ atoms $\left(N_{4}\right)$ goes from a broad feature $\left(N_{4}=0\right)$ corresponding to pure fermions, to the well structured one which is characteristic of bosons $\left(N_{4}=60\right)$. It is as if OCS were almost freely rotating inside the boson environment and, hence, these results were interpreted as a microscopic manifestation of superfluidity of ${ }^{4} \mathrm{He}$ at low temperatures. ${ }^{1}$

From the theoretical point of view, $\mathrm{Pi}$ et $a .^{2}{ }^{2}$ analyzed the structure of ${ }^{4} \mathrm{He} /{ }^{3} \mathrm{He}$ mixed drops around a dopant $\mathrm{SF}_{6}$ molecule or a $\mathrm{Xe}$ atom. In the framework of density functional theory, using a finite-range density functional, ${ }^{3}$ they found that the number of ${ }^{3} \mathrm{He}$ atoms, $N_{3}$, within the volume containing the first two solvation shells decreases when $N_{4}$ increases in such a way that the dopants may be already in a superfluid environment for $N_{4} \geqslant 60$. This is in good accord with path integral ${ }^{4}$ and variational ${ }^{5}$ Monte Carlo calculations. Also, path integral calculations on $\mathrm{SF}_{6}$ embedded in small

\footnotetext{
a) Author to whom correspondence should be addressed. Electronic mail: p.villarreal@imaff.cfmac.csic.es
}

${ }^{4} \mathrm{He}$ clusters ${ }^{6}$ revealed that superfluidity persists to some extent even in the highly compressed layers close to the dopant. In turn, Babichenko and Kagan ${ }^{7}$ attributed the strong distinction in the rotational structure of molecules in boson/fermion helium liquids to the difference in the spectral density of excitations, the large width of rotational levels in ${ }^{3} \mathrm{He}$ being determined by particle-hole excitations, whereas the small density of phonon excitations gives rise to the small broadening of levels in the ${ }^{4} \mathrm{He}$ case.

Jungwirth and $\operatorname{Krylov}^{8}$ incorporated the well established methodology of ab initio quantum chemistry, based on the analogy of fermionic $\mathrm{He}$ atoms and electrons, to study small ${ }^{3} \mathrm{He}_{N}(N=1,2)$ clusters doped with an atomic or molecular impurity. Heidenreich and Jortner extended the approach also to bosons and performed configuration interaction calculations of anthracene $\cdot \mathrm{He}_{N}(N=1,2)$ clusters. ${ }^{9}$ In the present work we follow an approach similar to that of Jungwirth and Krylov but considering larger fermionic and bosonic $\mathrm{He}$ clusters doped with a diatomic molecule. To this end, Hartree and Hartree-Fock calculations are performed within an adiabatic separation of the diatomic vibration, thereby providing energy levels and wave functions which allow us to simulate spectra of the perturbed diatomic molecule.

The paper is organized as follows: Sec. II briefly describes the simple model used to obtain energy levels of this kind of clusters resorting to Quantum Chemistry-type calculations. Then, the simulation of Raman spectra, appropriate for the case of homonuclear diatomic dopants, is outlined. Results stressing the different behavior of fermion/boson solvent helium atoms, as well as the case of mixtures, are presented in Sec. III. Finally, some conclusions and prospectives for future work are collected in Sec. IV. 


\section{THE THEORETICAL MODEL}

The basic equations for the approximate quantummechanical calculations are briefly presented. First, we describe the fundamental aspects of Quantum Chemistry-type treatments, i.e., Hartree or Hartree-Fock approaches which allows us to estimate initial and final wave-functions for the systems under study. These are in turn used to simulate vibrotational Raman spectra which clearly describe the very different behavior of bosonic versus fermionic statistics for the solvent atoms.

\section{A. Adiabatic approximation for pure bosonic/ fermionic species}

Using satellite coordinates $\left(\mathbf{r}, \mathbf{R}_{k}\right)$, where $\mathbf{r}$ is the vector joining the two bromine atoms and $\mathbf{R}_{k}$ are vectors from the diatomic center of mass to the different helium atoms, the Hamiltonian for the system under study can be approximated as

$$
\begin{aligned}
H^{\left(N_{\epsilon}\right)}= & -\frac{\hbar^{2}}{2 m} \frac{\partial^{2}}{\partial r^{2}}+U(r)+\frac{\mathbf{j}^{2}}{2 m r^{2}}+\sum_{k=1}^{N} h_{k}^{(\epsilon)}\left(\mathbf{R}_{k}, r\right) \\
& +\sum_{k<l} V_{k l}\left(\left|\mathbf{R}_{k}-\mathbf{R}_{l}\right|\right),
\end{aligned}
$$

where kinetic couplings, which are small for low-lying states, ${ }^{10}$ have been neglected. It consists of a diatomic Hamiltonian (the first three terms), plus $N$ three-atom $\mathrm{He}-\mathrm{Br}_{2}$ subsystems (fourth term) and the He-He interactions (fifth term). In Eq. (1), $m$ is the diatomic reduced mass, $\mathbf{j}$ is the angular momentum associated to $\mathbf{r}$, and $U$ represents the intramolecular diatomic potential. In turn, the Hamiltonian corresponding to each $\mathrm{He}-\mathrm{Br}_{2}$ subsystem is given by

$$
h_{k}^{(\epsilon)}\left(\mathbf{R}_{k}, r\right)=-\frac{\hbar^{2}}{2 \mu_{\epsilon}} \frac{\partial^{2}}{\partial R_{k}^{2}}+\frac{\mathbf{l}_{k}^{2}}{2 \mu_{\epsilon} R_{k}^{2}}+W\left(r, R_{k}, \theta_{k}\right)
$$

with $\mu_{\epsilon}$ being the reduced ${ }^{\epsilon} \mathrm{He}-\mathrm{Br}_{2}$ mass $(\epsilon=3,4), \mathbf{l}_{k}$ the angular momentum associated to $\mathbf{R}_{k}$, and $W$ the weak atomdiatom intermolecular potential depending on the $\left(r, R_{k}\right)$ distances and the orientation $\theta_{k}$, which is the angle formed by $\mathbf{r}$ and $\mathbf{R}_{k}$ vectors.

Choosing a body-fixed (BF) coordinate system with the $Z$ axis parallel to $\mathbf{r}$, we introduce a crude adiabatic approximation similar to that used to calculate vibrational frequency shifts ${ }^{11}$ in which, at different values of the intramolecular distance $r$, one looks for the ground state by solving Schrödinger equations of the type

$$
\left[\sum_{k=1}^{N} h_{k}^{(\epsilon)}+\sum_{k<l} V_{k l}-E_{\Lambda, S}^{(N)}(r)\right] \Phi_{\Lambda, S}^{(N)}\left(\left\{\mathbf{R}_{k}\right\} ; r\right)=0
$$

through either Hartree or Hartree-Fock methods depending on the helium isotope under consideration. In Eq. (3), the $r$-dependent eigenvalues, which are labeled by $\Lambda$, the projection of the orbital angular momentum $\mathbf{L}=\sum_{k=1}^{N_{\epsilon}} \mathbf{l}_{k}$ on the molecular axis, and the spin $S$, constitute an additional potential term for the diatomic molecule. Those levels, which in the bosonic scenario correspond to ${ }^{2 S+1} \Lambda={ }^{1} \Sigma$ states, are degenerate with respect to the spin projection $\Sigma$. For a total angular momentum $\mathbf{J}=\mathbf{j}+\mathbf{L}+\mathbf{S}$, with projection onto the BF $Z$-axis $\Omega=\Lambda+\Sigma$, the states of the "modified" diatom obey the Schrödinger equation

$$
\left[H_{N_{\epsilon}}^{\mathrm{eff}}-\varepsilon_{J S \Lambda \Sigma_{v}}\right] \chi_{J S \Lambda \Sigma_{v}}(r)=0,
$$

where $v$ is the stretching quantum number and the effective Hamiltonian, neglecting Coriolis couplings, can be expressed as

$$
H_{N_{\epsilon}}^{\mathrm{eff}}=-\frac{\hbar^{2}}{2 m} \frac{\partial^{2}}{\partial r^{2}}+U(r)+E_{\Lambda, S}^{(N)}(r)+\frac{\hbar^{2}}{2 m r^{2}} G,
$$

where, neglecting adiabatic corrections, $G$ is given by

$$
G=J(J+1)+\bar{L}(\bar{L}+1)+S(S+1)-2\left(\Lambda^{2}+\Sigma^{2}+\Lambda \Sigma\right) .
$$

Since the orbital angular momentum is not a good quantum number, an average

$$
\left\langle\mathbf{L}^{2}\right\rangle=\left\langle\Phi_{\Lambda, S}^{(N)}\left|\left(\sum_{k=1}^{N} \mathbf{I}_{k}\right)^{2}\right| \Phi_{\Lambda, S}^{(N)}\right\rangle \approx \hbar^{2} \bar{L}(\bar{L}+1)
$$

over $\Phi_{\Lambda, S}^{\left(N_{\epsilon}\right)}\left(\left\{\mathbf{R}_{k}\right\} ; r_{e q}\right)$ functions, estimated at a selected equilibrium distance $r_{e q}$, has been included. Note that, within the present approximation, the total wave function becomes

$$
\Psi \approx \mathcal{D}_{M \Omega}^{J^{*}}\left(\varphi_{r}, \theta_{r}, 0\right) \Phi_{\Lambda, S}^{\left(N_{\epsilon}\right)}\left(\left\{\mathbf{R}_{k}\right\} ; r\right) \chi_{J S \Lambda \Sigma v}(r),
$$

where $\mathcal{D}_{M \Omega}^{J}$ are Wigner rotation matrices that depend on the angular polar components $\left(\theta_{r}, \varphi_{r}\right)$ of $r$ in a space-fixed frame.

\section{B. Fermion/boson mixtures}

When one deals with $N$ fermion/ $M$ boson mixtures, a simple approach can be envisaged by starting at a zero-order level corresponding to the pure case, i.e., one solves Eq. (3) separately for $\left(E_{\Lambda, S}^{(N)}, \Phi_{\Lambda, S}^{(N)}\right)$, and $\left(E_{0,0}^{(M)}, \Phi_{0,0}^{(M)}\right)$. The effective diatomic Hamiltonian can be estimated through replacement of $E_{\Lambda, S}^{(N)}$ in Eq. (5) with a sum of the separate energies plus the first-order perturbation term coming from fermion-boson interactions, $V_{F / B}=\sum_{k=1}^{N} \Sigma_{l=1}^{M} V_{k l}$, i.e., one writes

$$
E_{\Lambda, S}^{(N / M)}(r)=E_{\Lambda, S}^{(N)}(r)+E_{0,0}^{(M)}(r)+\left\langle\left\langle V_{F / B}^{(N / M)}\right\rangle\right\rangle,
$$

where $\left\langle\left\langle V_{F / B}^{(N / M)}\right\rangle\right\rangle=\left\langle\Phi_{\Lambda, S}^{(N)} \Phi_{0,0}^{(M)}\left|V_{F / B}\right| \Phi_{0,0}^{(M)} \Phi_{\Lambda, S}^{(N)}\right\rangle$. Note that now $\mathbf{L}=\sum_{k=1}^{N} \mathbf{l}_{k}+\sum_{j=1}^{M} \mathbf{l}_{j}$ and $\left\langle\mathbf{L}^{2}\right\rangle$ must be recalculated.

One further resorts to a SCF treatment ${ }^{12}$ in which the wave function is expressed as a product $\Phi_{\Lambda, S}^{(N)} \Phi_{0,0}^{(M)}$ where the factors are modified using now iterative Hartree-Fock/ Hartree calculations up to convergence,

$$
\begin{aligned}
& {\left[\sum_{k=1}^{N} h_{k}^{(3)}+\sum_{k<l}^{N} V_{k l}+V_{F / B}^{(M)}-\widetilde{E}_{\Lambda, S}^{(N)}(r)\right] \Phi_{\Lambda, S}^{(N)}=0,} \\
& {\left[\sum_{k=1}^{M} h_{k}^{(4)}+\sum_{k<l}^{M} V_{k l}+V_{F / B}^{(N)}-\widetilde{E}_{0,0}^{(M)}(r)\right] \Phi_{0,0}^{(M)}=0,}
\end{aligned}
$$

where $V_{F / B}^{(M)}$ and $V_{F / B}^{(N)}$ stand for the averages $\left\langle\Phi_{0,0}^{(M)}\left|V_{F / B}\right| \Phi_{0,0}^{(M)}\right\rangle$ and $\left\langle\Phi_{\Lambda, S}^{(N)}\left|V_{F / B}\right| \Phi_{\Lambda, S}^{(N)}\right\rangle$, respectively. 
The corresponding energy becomes now

$$
\widetilde{E}_{\Lambda, S}^{(N / M)}(r)=\widetilde{E}_{\Lambda, S}^{(N)}(r)+\widetilde{E}_{0,0}^{(M)}(r)-\left\langle\Phi_{\Lambda, S}^{(N)}\left|V_{F / B}^{(M)}\right| \Phi_{\Lambda, S}^{(N)}\right\rangle .
$$

By subtracting to $\widetilde{E}_{\Lambda, S}^{(N / M)}$ the zero-order separated energies, an effective coupling similar to that of Eq. (9) can be defined,

$$
\left\langle\left\langle V_{F / B}^{(N / M)}\right\rangle\right\rangle=\widetilde{E}_{\Lambda, S}^{(N / M)}(r)-E_{\Lambda, S}^{(N)}(r)-E_{0,0}^{(M)}(r) .
$$

\section{Hartree-Fock and Hartree expansions}

As mentioned above, in order to solve Eq. (3) we used Hartree-Fock or Hartree approaches depending on the helium isotope under consideration. In particular, the nuclear wave function of doped ${ }^{3} \mathrm{He}$ clusters is approximated as a Slater determinant, i.e. as an antisymetrized product of one-fermion spin-orbitals,

$$
\begin{aligned}
\Phi_{\Lambda, S}^{(N)}= & \frac{1}{\sqrt{N !}} \operatorname{det}\left[\phi_{1}\left(\mathbf{R}_{1}, s_{1} ; r\right) \ldots \phi_{i}\left(\mathbf{R}_{i}, s_{i} ; r\right) \ldots\right. \\
& \left.\times \phi_{N}\left(\mathbf{R}_{N}, s_{N} ; r\right)\right]
\end{aligned}
$$

with $s_{i}(i=1, \ldots, N)$ denoting the spin coordinates of the $N$ fermions. In turn, each one-fermion wave function $\phi_{i}\left(\mathbf{R}_{i}, \mathbf{s}_{i} ; r\right)$ is a product of a spatial one-particle orbital $\psi_{i}\left(\mathbf{R}_{i} ; r\right)$ and a spin function $\sigma\left(\mathbf{s}_{i}\right)=\alpha\left(\mathbf{s}_{i}\right)$ or $\beta\left(\mathbf{s}_{i}\right)$. The spatial one-particle orbitals are found through the restricted closed-shell or open-shell Hartree-Fock methods, depending of the spin-symmetry of the system, by using a direct minimization procedure ${ }^{13}$ to force convergence to the global minimum. For a singlet spin-symmetry state $(S=0)$, the wave function is explicitly given by

$$
\begin{aligned}
\Phi_{\Lambda, 0}^{(N)}= & \frac{1}{\sqrt{N !}} \operatorname{det}\left[\psi_{1}\left(\mathbf{R}_{1} ; r\right) \alpha\left(s_{1}\right) \psi_{1}\left(\mathbf{R}_{2} ; r\right) \beta\left(s_{2}\right) \ldots\right. \\
& \left.\times \psi_{N / 2}\left(\mathbf{R}_{N} ; r\right) \alpha\left(s_{N}\right) \psi_{N / 2}\left(\mathbf{R}_{N} ; r\right) \beta\left(s_{N}\right)\right]
\end{aligned}
$$

and, for a general spin-symmetry state with $(2 S+1)$ multiplicity, it is written as

$$
\begin{aligned}
\Phi_{\Lambda, S}^{(N)}= & \frac{1}{\sqrt{N !}} \operatorname{det}\left[\psi_{1}\left(\mathbf{R}_{1} ; r\right) \alpha\left(s_{1}\right) \psi_{1}\left(\mathbf{R}_{2} ; r\right) \beta\left(s_{2}\right) \ldots\right. \\
& \times \psi_{N / 2-S}\left(\mathbf{R}_{N-2 S-1} ; r\right) \alpha\left(\mathbf{s}_{N-2 S-1}\right) \\
& \times \psi_{N / 2-S}\left(\mathbf{R}_{N-2 S} ; r\right) \beta\left(\mathbf{s}_{N-2 S}\right) \ldots \\
& \left.\times \psi_{N / 2-S-1}\left(\mathbf{R}_{N-1} ; r\right) \alpha\left(s_{N-1}\right) \psi_{N / 2-S}\left(\mathbf{R}_{N} ; r\right) \alpha\left(s_{N}\right)\right] .
\end{aligned}
$$

On the other hand, the nuclear wave function of doped ${ }^{4} \mathrm{He}$ clusters is taken as a Hartree product in which all the bosons are assumed to occupy the same one-particle orbital,

$$
\Phi_{\Lambda, 0}^{\left(N_{4}\right)}=\psi_{1}\left(\mathbf{R}_{1} ; r\right) \ldots \psi_{1}\left(\mathbf{R}_{i} ; r\right) \ldots \psi_{1}\left(\mathbf{R}_{N} ; r\right) .
$$

For bosons and singlet fermions, and starting from $N$ $=2$, the initial orbitals are those corresponding to independent particles. Here on, the chosen initial orbitals were those obtained from the preceding calculation with $N-2$ particles. For $S \neq 0$ fermions, one starts with the optimized orbitals from the $S-1$ calculation for the same size cluster. In either case, spatial one-particle orbitals entering Eqs. (14)-(16) are expanded in terms of a finite basis set composed of products of radial and angular functions,

$$
\chi^{(n l m)}(\mathbf{R} ; r)=g_{n}(R ; r) Y_{l m}(\theta, \phi),
$$

where $Y_{l m}(\theta, \phi)$ are spherical harmonics. The radial $g_{n}(R ; r)$ basis functions are obtained by solving for the ground level the Schrödinger equation corresponding to the triatomic ${ }^{\epsilon} \mathrm{He}-\mathrm{Br}_{2}$ subsystem at fixed orientations $\theta_{n}$,

$$
\left[-\frac{\hbar^{2}}{2 \mu_{\epsilon}} \frac{\partial^{2}}{\partial R^{2}}+W\left(R, \theta_{n} ; r\right)-E_{n}(r)\right] g_{n}\left(R, \theta_{n} ; r\right)=0,
$$

repeating the calculations for $n_{\max }$ different equidistant values of $\theta_{n}$ in the range [0, $\left.\pi / 2\right]$, and then using an orthogonalization Schmidt procedure among different $g_{n}\left(R, \theta_{n} ; r\right)$ radial functions.

\section{Vib-rotational Raman spectra simulation}

The aim is here to compute cross section profiles for a process of the type

$$
\mathrm{Br}_{2}-X_{N}(i)+\hbar \omega_{0} \rightarrow \mathrm{Br}_{2}-X_{N}(f)+\hbar \omega_{f i},
$$

where a photon of frequency $\omega_{0}$ induces on the polarizable system, in an initial state $|i\rangle$, an electric dipole which, in turn, may emit a photon of frequency $\omega_{f i}$ while the system emerges in a final state $|f\rangle$.

\section{1. "Stick" spectra}

Making use of the diatomic polarizability diagonal matrix in the BF frame, $\boldsymbol{\alpha}^{(\mathrm{BF})}$ (supposed to be unaffected by complexation) and of the polarization vector of the incident electric field $\underset{\mathbf{e}}{\mathbf{e}}$, the induced dipole moment can be written in the SF frame of reference with the help of first-rank rotation matrices $\mathbf{R}$

$$
\boldsymbol{\mu}={\underset{\sim}{\boldsymbol{\alpha}}}^{(\mathrm{SF})} \underset{\sim}{\hat{\mathbf{e}}}={\underset{\sim}{\mathbf{R}}}^{-1}{\underset{\sim}{\boldsymbol{\alpha}}}^{(\mathrm{BF})} \underset{\sim}{\mathbf{R}} \hat{\mathbf{e}}
$$

with spherical components

$$
\mu_{k}=\sum_{l} e_{l}^{*} \sum_{m} \mathcal{D}_{m k}^{1^{*}}\left(\varphi_{r}, \theta_{r}, 0\right) \alpha_{m} \mathcal{D}_{m l}^{1}\left(\varphi_{r}, \theta_{r}, 0\right) .
$$

By assuming linearly polarized incident light with its electric vector defining the space-fixed $Z$ direction and that propagates along the $Y$ axis (while the scattered light is detected along the $X$ axis) the components of the induced dipole become

$$
\begin{aligned}
\mu_{k} \sim \sum_{m}(-1)^{m} \alpha_{m} \sum_{n}(2 n+1)\left(\begin{array}{ccc}
1 & 1 & n \\
-m & m & 0
\end{array}\right) \\
\times\left(\begin{array}{ccc}
1 & 1 & n \\
-k & 0 & k
\end{array}\right) \mathcal{D}_{0-k}^{n}\left(\varphi_{r}, \theta_{r}, 0\right) .
\end{aligned}
$$

By carrying out an integral over three Wigner matrices (note the absence of the azimuthal variable) 


$$
\begin{aligned}
\int d \tau & \mathcal{D}_{M_{f} \Omega_{f}}^{J_{f}} \mathcal{D}_{0-k}^{n} \\
= & 4 \pi \delta_{M_{i} M_{f}}(-1)^{M_{i}}{ }_{M_{i} \Omega_{i}}^{J_{i}^{*}}\left(\begin{array}{ccc}
J_{i} & n & J_{f} \\
-M_{i} & 0 & M_{i}
\end{array}\right) \\
& \times\left(\begin{array}{ccc}
J_{i} & n & J_{f} \\
-\Omega_{i} & -k & \Omega_{f}
\end{array}\right),
\end{aligned}
$$

the matrix elements of the induced dipole moment components between initial and final states of type (8), $\mu_{k}^{f, i}$ $=\left\langle\Psi_{f}\left|\mu_{k}\right| \Psi_{i}\right\rangle$, can be estimated as

$$
\begin{aligned}
\mu_{k}^{f, i} \sim & \delta_{\Lambda_{f} \Lambda_{i}} \delta_{S_{f} S_{i}}(-1)^{M_{i}} \sum_{m, n}(-1)^{m} \alpha_{m}^{f, i}(2 n+1) \\
& \times\left(\begin{array}{ccc}
1 & 1 & n \\
-m & m & 0
\end{array}\right)\left(\begin{array}{ccc}
1 & 1 & n \\
-k & 0 & k
\end{array}\right)\left(\begin{array}{ccc}
J_{i} & n & J_{f} \\
-M_{i} & 0 & M_{i}
\end{array}\right) \\
& \times\left(\begin{array}{ccc}
J_{i} & n & J_{f} \\
-\Omega_{i} & -k & \Omega_{f}
\end{array}\right),
\end{aligned}
$$

where $\alpha_{m}^{f, i}=\left\langle\chi_{J_{f} S_{f} \Omega_{f} v_{f}}\left|\alpha_{m}\right| \chi_{J_{i} S_{i} \Omega_{i} v_{i}}\right\rangle$. Note that in the boson scenario $\left(\Omega_{i}=\Omega_{f}=0\right)$, the only surviving component is $\mu_{0}$, and the scattered light also emerges polarized along $Z$ (parallel signal). In this case, the sum over $n$ runs only over the values 0 and 2, giving rise to standard diatomiclike selection rules $\Delta J=0, \pm 2$, which contribute to the $Q$ and $O, S$ branches in the spectrum. In order to stress the difference of boson/fermion frameworks, we will consider here detection along the $X$ axis for linear, parallel polarization.

In terms of the spherical and the anisotropic parts of the polarizability, ${ }^{14}$ which in this case become $\alpha_{f, i}=\left(\alpha_{0}^{f, i}\right.$ $\left.+2 \alpha_{1}^{f, i}\right) / 3$ and $\beta_{f, i}^{2}=\left(\alpha_{0}^{f, i}-\alpha_{1}^{f, i}\right)^{2}$, respectively, and taking into account that $\alpha_{-1}^{f, i}=\alpha_{1}^{f, i}$ one finds,

$$
\begin{aligned}
\mu_{0}^{f, i} \sim \alpha_{f, i}\left(\begin{array}{ccc}
J_{i} & 0 & J_{f} \\
-M_{i} & 0 & M_{i}
\end{array}\right)\left(\begin{array}{ccc}
J_{i} & 0 & J_{f} \\
-\Omega_{i} & 0 & \Omega_{f}
\end{array}\right) \\
+\frac{2 \beta_{f, i}}{3}\left(\begin{array}{ccc}
J_{i} & 2 & J_{f} \\
-M_{i} & 0 & M_{i}
\end{array}\right)\left(\begin{array}{ccc}
J_{i} & 2 & J_{f} \\
-\Omega_{i} & 0 & \Omega_{f}
\end{array}\right) .
\end{aligned}
$$

The third component of $J$ is conserved in $\mathrm{SF}(\Delta M=0)$ as well as $\mathrm{BF}(\Delta \Omega=0)$ frames. We immediately see remarkable changes in the complexes containing fermions with $\Omega_{i}$ $=\Omega_{f} \neq 0$, since transitions with $\Delta J= \pm 1$ become allowed and, thus, $P$ and $R$ branches [with the exception of $P(1)$ and $R(0)]$ should appear in the spectrum.

For a fixed energy of the incident photon we can introduce a Boltzmann distribution over cluster states at a given temperature $T$ and average over initial rotational states. Hence, a line of intensity

$$
I_{f i}(T) \propto \frac{e^{-\left(\varepsilon_{i} / k T\right)}}{\sum_{i} e^{-\left(\varepsilon_{i} / k T\right)}} \frac{1}{2 J_{i}+1} \sum_{M_{i}}\left|\mu_{0}^{f, i}\right|^{2}
$$

would appear at an energy $\hbar \omega_{f i}=\hbar \omega_{0}-\left(\varepsilon_{J_{f}} S_{i} \Lambda_{i} \Sigma_{i} v_{f}\right.$ $-\varepsilon_{J_{i} S_{i} \Lambda_{i} \Sigma_{i} v_{i}}$ ) of the scattered photon. In Eq. (24), we have replaced $\varepsilon_{J_{i} S_{i} \Lambda_{i} \Sigma_{i} v_{i}}$ by $\varepsilon_{i}$ for simplicity of notation.

\section{Continuum spectra}

We turn now to providing a way of dressing the stick lines. If we consider the case in which the diatomic subunit is promoted to a vibrationally excited state, it can transfer the excess of energy to some of the weak bonds thereby causing break up of the complex. It is extremely difficult to appropriately estimate the broadening induced in the spectrum lines by this mechanism of vibrational predissociation (VP), but a reasonable modeling of the process may, however, be enough for our present purposes. After our previous dealings with VP for three-atomic. ${ }^{15}$ and tetraatomic ${ }^{16,17}$ species, the idea is to extend the treatment to larger systems accounting for two additional facts: (1) Different cluster sizes show different angular arrangements of the rare gas atoms with respect to the diatomic molecule, thus affecting the corresponding VP width, and (2) at least for moderate cluster sizes, the VP width of a system containing $N$ weak bonds should not be very different from $N$ times the width corresponding to $N=1$, though it should tend to some other limiting value as $N$ increases. So, our approximation starts with the calculation of angular-dependent VP rates $\Gamma_{v_{f} \leftarrow v_{i}}^{\epsilon}(\theta),{ }^{18}$ $\epsilon=3,4$, for the two three-atom ${ }^{3} \mathrm{He}$ - and ${ }^{4} \mathrm{He}-\mathrm{Br}_{2}$ species at $J=0$ (slightly different values are obtained for $J \neq 0$ ). They are being averaged over angular distributions $\mathcal{D}_{\Lambda, S}^{\left(N_{\epsilon}\right)}(\theta)$ coming from our previous $\Phi_{\Lambda, S}^{\left(N_{\epsilon}\right)}\left(\left\{\mathbf{R}_{k}\right\} ; r_{e q}\right)$ functions, Eq. (3). In addition, with regard to the second requirement of above, the number of effective bonds [estimated via the relation $N_{\epsilon}^{\text {eff }}$ $\left.=E_{\Lambda, S}^{\left(N_{\epsilon}\right)}\left(r_{e q}\right) / E^{\left(1_{\epsilon}\right)}\left(r_{e q}\right)\right]$ instead of a pure $N_{\epsilon}$ dependence, is accounted for. The VP rate is finally given by

$$
\Gamma_{v_{f} \leftarrow v_{i}}^{\left(N_{\epsilon}\right)}=N_{\epsilon}^{\mathrm{eff}} \int_{0}^{\pi} d \theta \mathcal{D}_{\Lambda, S}^{\left(N_{\epsilon}\right)}(\theta) \Gamma_{v_{f} \leftarrow v_{i}}^{\epsilon}(\theta) .
$$

Note that for simplicity, the required energies and wave functions were those corresponding to the diatomic equilibrium distance, see Eq. (3).

The previous estimate applies to pure bosonic or fermionic complexes. When dealing with a mixture of $N$ fermions and $M$ bosons, one can "democratically" share the coupling term, Eq. (12), between the two components, getting effective numbers of fermionic or bosonic bonds,

$$
\begin{aligned}
& \mathcal{N}_{3}=\left(E_{\Lambda, S}^{\left(N_{3}\right)}\left(r_{e q}\right)+\frac{N}{M+N}\left\langle\left\langle V_{F / B}^{N / M}\right\rangle\right\rangle\right) / E^{\left(1_{3}\right)}\left(r_{e q}\right) \\
& \mathcal{M}_{4}=\left(E^{\left(M_{4}\right)}\left(r_{e q}\right)+\frac{M}{M+N}\left\langle\left\langle V_{F / B}^{N / M}\right\rangle\right\rangle\right) / E^{\left(1_{4}\right)}\left(r_{e q}\right)
\end{aligned}
$$

and the corresponding VP rate therefore becomes

$$
\Gamma_{v_{f} \leftarrow v_{i}}^{\left(N_{3} / M_{4}\right)}=\frac{\mathcal{N}_{3}}{N_{3}^{\text {eff }}} \Gamma_{v_{f} \leftarrow v_{i}}^{\left(N_{3}\right)}+\frac{\mathcal{M}_{4}}{M_{4}^{\text {eff }}} \Gamma_{v_{f} \leftarrow v_{i}}^{\left(M_{4}\right)}
$$

Eq. (26) constitutes a generalization of Eq. (25) and recovers the original result for pure clusters, i.e., for $N=0$ or $M=0$.

By dressing the corresponding lines with Lorentzian functions and by summing over transitions, a continuum spectrum is obtained,

$$
\sigma(\omega ; T)=\sum_{f, i} \frac{\Gamma_{f i} / 2 \pi}{\hbar^{2}\left(\omega-\omega_{f, i}\right)^{2}+\Gamma_{f i}^{2} / 4} I_{f i}(T)
$$


which satisfies the condition

$$
\int d \omega \sigma(\omega ; T)=\sum_{f, i} I_{f i}(T) .
$$

\section{RESULTS}

In the calculations presented here the following masses (amu) were used: $m_{\mathrm{Br}}=78.91830, m^{m_{3} \mathrm{He}}=3.01604$, and $m_{4}{ }_{\mathrm{He}}=4.00260$. A grid of 5000 points in the range [1.518.5] A was employed to numerically solve Eq. (18), using a Numerov procedure, at different orientations for fixed bromine bond-length distances of $r=2.20,2.281$, and $2.35 \AA$, where Quantum Chemistry calculations were performed. Convergence in the fermionic cases was achieved by using $l_{\max }=8,\left|m_{\max }\right|=3$, and $n_{\max }=4$, see Eqs. (17) and (18), and $l_{\max }=12,\left|m_{\max }\right|=1$, and $n_{\max }=4$ for bosons. With the above basis sets, the needed one-particle and two-particle integrals were evaluated analytically in the angular variables and numerically in the radial ones. ${ }^{10}$

\section{A. Interaction potentials}

The $\mathrm{Br}_{2}(X)$ intermolecular interaction $U$ was described by a Morse function, ${ }^{19}$

$$
U(r)=D\left\{1-\exp \left[-\alpha\left(r-r_{e q}\right)\right]\right\}^{2}
$$

while the ${ }^{\epsilon} \mathrm{He}-\mathrm{Br}_{2}(X)$ intramolecular potential $W$ was approximated as a pairwise addition of Morse $\mathrm{He}-\mathrm{Br}$ interactions in the ground state, ${ }^{20}$

$$
W(r, R, \theta)=M\left(R_{1}\right)+M\left(R_{2}\right),
$$

where $R_{i}, i=1,2$ are the two He-Br distances and

$$
M\left(R_{i}\right)=D\left\{1-\exp \left[-\alpha\left(R_{i}-R_{e q}\right)\right]\right\}^{2}-D .
$$

In turn, the He-He interaction $V$ was modeled as a Morse potential from Ref. 21 when carrying out diffusion Monte Carlo (DMC) calculations. Because of the mean-field nature of the Hartree and Hartree-Fock treatments based on oneparticle orbitals, the He-He repulsion at short distances is overestimated. $^{22}$ In fact, for two bosons, and two fermions in a spin state $S=0$, the potential of Ref. 21 gives binding energies of only $\sim 26 \mathrm{~cm}^{-1}$ and $\sim 19 \mathrm{~cm}^{-1}$, respectively. These values are to be compared with the corresponding accurate variational ${ }^{10}$ estimates of $36.28 \mathrm{~cm}^{-1}$ and $30.71 \mathrm{~cm}^{-1}$. As the systems increase in size they become artificially unbound and Hartree/Hartree-Fock calculations using the original He-He interaction ${ }^{21}$ become meaningless. This difficulty is overcome by introducing an effective truncated core potential $^{3,22,23} V \exp [-\gamma V]$ to better account for short-range correlation. The $\gamma$ parameter selects the maximum allowed interaction at short distances, and it was adjusted to get good agreement with the "exact" DMC energies for bosons.

The more accurate description of the short range correlation, formulated for pairs of bosonic $\mathrm{He}$ atoms and achieved through introduction of an effective truncated core potential, also applies to pairs of fermionic He atoms in the singlet spin multiplicity state, as the latter are not different, apart from the mass, from the former. Thus the lowest spinmultiplicity state, which is the most correlated, is in fact also
TABLE I. Parameters of different atom-atom interactions.

\begin{tabular}{ccccc}
\hline \hline Interaction & $D\left(\mathrm{~cm}^{-1}\right)$ & $\alpha\left(\AA^{-1}\right)$ & $\rho_{e q}(\AA)$ & $\gamma\left(\mathrm{au}^{-1}\right)$ \\
\hline $\operatorname{Br}_{2}(X)$ & 24557.674 & 1.588 & 2.281 & \\
$\mathrm{He}-\mathrm{Br}(X)$ & 19.62 & 1.55 & 3.81 & \\
$\mathrm{He}-\mathrm{He}$ & 7.61 & 2.126 & 2.963 & 2000 \\
\hline \hline
\end{tabular}

corrected. In this way, the improved description of the short range ("intrashell") correlation carries over to the high multiplicity states as well. Therefore the same value of $\gamma$ was used for all pairs of He atoms irrespective of their bosonic or fermionic character. Table I collects the values of $\gamma$ and the rest of parameters used to describe the different potentials for atom-atom interactions.

\section{B. Energetics}

For pure bosonic or fermionic $(S=0)$ species, with the $\mathrm{Br}-\mathrm{Br}$ distance at its equilibrium value, $r=2.281 \AA$, Fig. 1 displays the evolution of ground state energies with the size, i.e., the number $N$ of $\mathrm{He}$ atoms in the cluster. DMC calculations $^{24}$ are included in order to show the accuracy of Hartree results along the whole range of sizes considered. The truncated core parameter $\gamma$ of the scaled He-He potential was fitted at $N=2$. A smooth increase of binding energies with the size can be seen for the two kinds of isotopic species, suggesting the tendency to a limiting value. The limited cluster size considered in this work does not allow as yet to reach such a value. We further see lower binding energies for fermionic than bosonic clusters, a feature of the combined consequence of the lighter mass of ${ }^{3} \mathrm{He}$ and the spin effect, which imposes the filling of excited spatial orbitals for fermionic statistics.

Figure 2 depicts the corresponding effective potentials of Eq. (5) in the neighborhood of the $\mathrm{Br}_{2}$ equilibrium distance and together with the diatomic interaction. In the fermion and the mixture cases, the degree of energy degeneracy does not allow to distinguish among different multiplicities within the scale of the figure, so only the singlet, $S=0$, cases are included. In spite of the fact that the effective potentials ap-

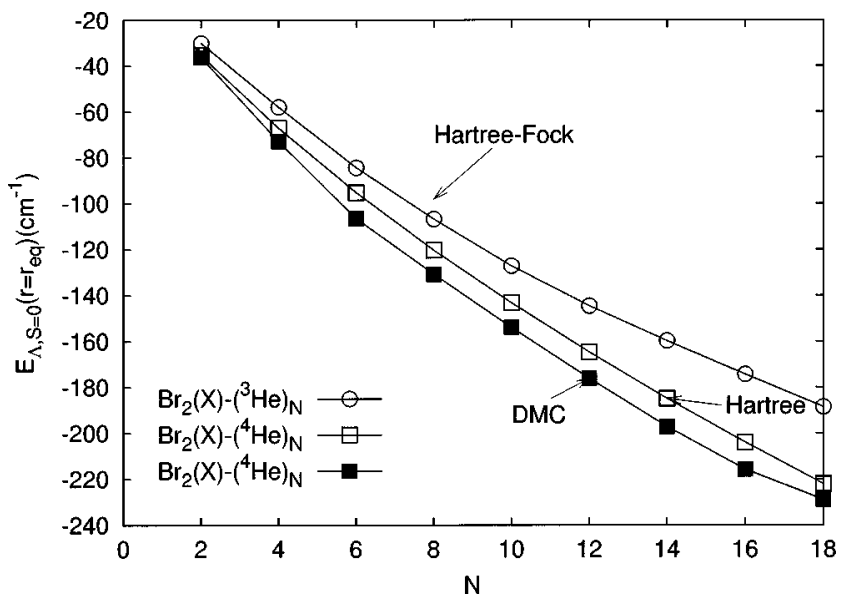

FIG. 1. Ground energies of complexes as a function of the number of $\mathrm{He}$ atoms, calculated at the equilibrium $\mathrm{Br}_{2}$ bond-length, $r_{e q}=2.281 \AA$. For ${ }^{4} \mathrm{He}$, Hartree calculations are compared with "exact" DMC ones. For ${ }^{3} \mathrm{He}$, Hartree-Fock singlet state $(S=0)$ levels are reported. 


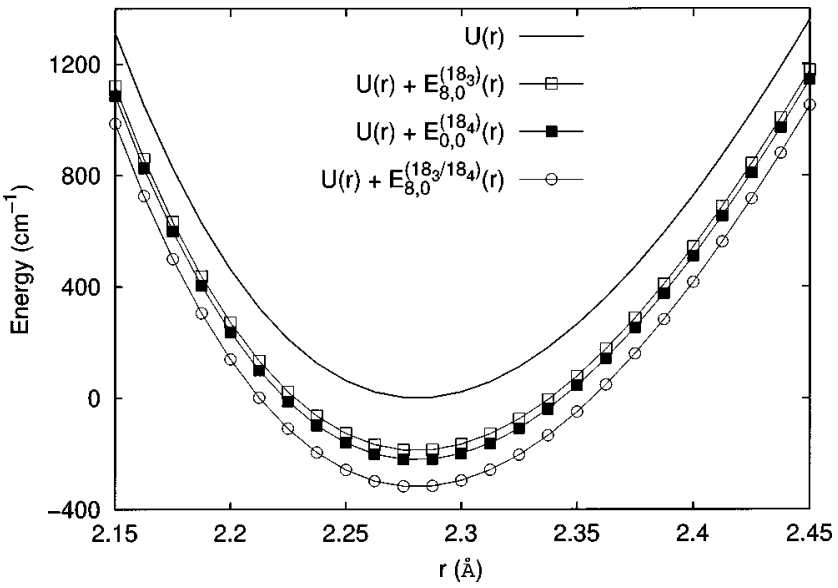

FIG. 2. Effective potentials as functions of the $\mathrm{Br}_{2}(X)$ bond length for complexes containing $18{ }^{3} \mathrm{He}$ atoms (singlet state), $18{ }^{4} \mathrm{He}$ atoms, and a mixture of $18{ }^{3} \mathrm{He}$ and $18{ }^{4} \mathrm{He}$ atoms (singlet state), together with the potential for the isolated diatom.

pear to be a simple translation from the "bare" diatomic interaction, the additional terms show a slow linear dependence on $r$, with decreasing values as $r$ increases. For the vibrational shifts we can compare the $v=0 \rightarrow 1$ vibrational spacing at $J=0$ for the isolated diatomic molecule with the three clusters which contain 18 fermion, 18 boson, and a mixture of ( 18 fermion +18 boson) He atoms, solving Eq. (4). In $\mathrm{Br}_{2}(X)$ alone this quantity $\left(\mathrm{cm}^{-1}\right)$ is 323.1561 . It increases smoothly to $323.3452(18 \mathrm{~F}, S=3,4), 323.3554$ $(18 \mathrm{~B})$, and $323.5396(18 \mathrm{~F} / 18 \mathrm{~B}, S=3)$. As for the rotational shift, i.e., $J=0 \rightarrow 1$ at $v=0$, within an error of $0.0001 \mathrm{~cm}^{-1}$, there is no variation with respect to the diatomic value of $0.1639 \mathrm{~cm}^{-1}$.

At the equilibrium $\mathrm{Br}_{2}(X)$ bond distance and for the species containing 18 fermions, 18 bosons and a mixing of them, energies, mean values of the squared orbital angular momenta and their projection on the intermolecular axis are listed in Table II. The role played by the spin multiplicity becomes apparent since the complexes with fermions show a density of levels which is in contrast with the single level appearing for the bosonic case. For pure fermions, and up to $S=5$, the different levels show up within an energy range smaller than $3 \mathrm{~cm}^{-1}$, which has profound effects on the cor-
TABLE III. Convergence study, through the SCF procedure described in Sec. II B, of the energy at $r=r_{\text {eq }}$ corresponding to different multiplets in the mixed complex of 18 fermions/18 bosons.

\begin{tabular}{cccc}
\hline \hline $2 S+1$ & $(0)$ & $(1)$ & $(2)$ \\
\hline 1 & -286.03 & -318.69 & -318.70 \\
3 & -286.72 & -319.07 & -318.99 \\
5 & -287.91 & -318.09 & -319.71 \\
7 & -294.46 & -324.24 & -327.40 \\
9 & -290.23 & -321.09 & -321.29 \\
11 & -293.41 & -321.02 & -319.42 \\
13 & -295.36 & -321.09 & -323.10 \\
15 & -293.17 & -323.99 & -327.05 \\
17 & -293.55 & -320.62 & -323.36 \\
19 & -293.59 & -319.33 & -322.80 \\
\hline \hline
\end{tabular}

responding Raman spectra, as will be shown later. They exhibit a broad distribution of $\Lambda$ values, although the lowest one $(S=4)$ corresponds to a $\Sigma$ state. In spite of the high degree of degeneracy shown by the mixture, the lowest level, the $S=\Lambda=3$ state, is found far from the rest of them and close to the $S=7, \Lambda=1$ level.

For the mixture containing 18 fermions and 18 bosons, and with the bromine bond fixed at $r_{e q}$, Table III lists the set of energy values for the different spin multiplicities, obtained by applying the procedure described in Sec. II B, up to a second step. When one compares the results of the last row with those of the previous one, a degree of convergence better than $1 \%$ can be seen. Although the zero-order level (which coincides with the first order of perturbation) is far from having reached convergence, the distribution of energies is maintained along the process with the single exception of the $S=6$ state that is the lowest one at this level. On the contrary, a first alternate Hartree/Hartree-Fock calculation leads quickly to converged results.

In order to perform spectra simulations, we carried out calculations at different values of the bromine bond-length. Table IV collects the results obtained for the three cluster sizes already mentioned, and for the different spin multiplicities, at two additional values of $r, 2.20$ and $2.35 \AA$, covering the $v=1$ level of the isolated bromine. Within this range, a linear decrease of binding energies as $r$ increases is found for every complex and at all multiplicities. So, by adding these

TABLE II. Energies, mean value of the squared orbital angular momentum, and its projection on the interdiatomic axis of boson, fermion and mixture complexes. They are calculated at the equilibrium $\operatorname{Br}_{2}(X)$ bond distance and depend on the spin multiplicity.

\begin{tabular}{|c|c|c|c|c|c|c|c|c|c|}
\hline \multirow[b]{2}{*}{$2 S+1$} & \multicolumn{3}{|c|}{$\left({ }^{4} \mathrm{He}\right)_{18}$} & \multicolumn{3}{|c|}{$\left({ }^{3} \mathrm{He}\right)_{18}$} & \multicolumn{3}{|c|}{$\left({ }^{4} \mathrm{He}\right)_{18}+\left({ }^{3} \mathrm{He}\right)_{18}$} \\
\hline & $\mathrm{E}$ & $\left\langle L^{2}\right\rangle$ & $\Lambda$ & E & $\left\langle L^{2}\right\rangle$ & $\Lambda$ & $\mathrm{E}$ & $\left\langle L^{2}\right\rangle$ & $\Lambda$ \\
\hline 1 & -222.17 & 66.42 & 0 & -188.40 & 111.57 & 8 & -318.70 & 230.74 & 8 \\
\hline 3 & & & & -188.90 & 84.44 & 6 & -318.99 & 205.73 & 6 \\
\hline 5 & & & & -189.84 & 105.74 & 7 & -319.71 & 230.45 & 7 \\
\hline 7 & & & & -190.25 & 75.94 & 3 & -327.40 & 203.15 & 3 \\
\hline 9 & & & & -191.11 & 69.96 & 0 & -321.29 & 187.32 & 0 \\
\hline 11 & & & & -188.73 & 64.64 & 0 & -319.42 & 240.54 & 0 \\
\hline 13 & & & & -185.80 & 55.86 & 0 & -323.10 & 233.99 & 0 \\
\hline 15 & & & & -180.76 & 34.18 & 1 & -327.05 & 237.33 & 1 \\
\hline 17 & & & & -175.26 & 49.21 & 2 & -323.36 & 255.57 & 2 \\
\hline 19 & & & & -169.31 & 57.50 & 0 & -322.80 & 276.14 & 0 \\
\hline
\end{tabular}


TABLE IV. Variation of the energy $\left(\mathrm{cm}^{-1}\right)$ with the $\mathrm{Br}_{2}$ bond length $r(\AA)$ of the different multiplets in the complexes considerated. Entries correspond to the sequence $r=2.20 / 2.281 / 2.35$.

\begin{tabular}{cccc}
\hline \hline $2 S+1$ & $\left({ }^{4} \mathrm{He}\right)_{18}$ & $\left({ }^{3} \mathrm{He}\right)_{18}$ & $\left({ }^{4} \mathrm{He}\right)_{18}+\left({ }^{3} \mathrm{He}\right)_{18}$ \\
\hline 1 & $-225.06 /-222.17 /-219.76$ & $-191.21 /-188.40 /-186.08$ & $-323.66 /-318.70 /-314.55$ \\
3 & & $-191.74 /-188.90 /-186.58$ & $-325.98 /-319.00 /-314.74$ \\
5 & $-192.61 /-189.84 /-187.54$ & $-325.02 /-319.71 /-314.19$ \\
7 & $-192.99 /-190.25 /-187.98$ & $-333.14 /-327.40 /-322.57$ \\
9 & $-193.82 /-191.11 /-188.86$ & $-326.91 /-321.29 /-314.15$ \\
11 & $-191.43 /-188.73 /-186.49$ & $-324.52 /-319.42 /-314.97$ \\
13 & $-188.51 /-185.80 /-183.55$ & $-327.95 /-323.10 /-319.43$ \\
15 & $-183.53 /-180.77 /-178.50$ & $-332.00 /-327.05 /-320.69$ \\
17 & $-178.01 /-175.26 /-173.00$ & $-326.29 /-323.36 /-319.38$ \\
19 & $-172.07 /-169.31 /-167.06$ & $-327.94 /-322.80 /-318.52$ \\
\hline \hline
\end{tabular}

energies to the diatomic potential, Eq. (5), a decrease of the equilibrium value would be expected when going from the isolated diatom to the different complexes, leading to a lowering of the moments of inertia. This is in clear disagreement with the experimental facts ${ }^{1}$ which, for boson complexes and according to a classical model, correspond to moments of inertia equal to that of the diatomic molecule plus the contribution of the nearest helium atoms and the additional hydrodynamic terms. However, one must realize that for high values of the orbital angular momentum $\bar{L}$, the centrifugal term in Eq. (5) may dominate over the additional potential term, so the combined effect may go in the reverse order, i.e., in agreement with the experiment. For the largest boson cluster considered, we estimate that this effect would take place for $\bar{L}>22$. This quantity increases with the size of the cluster, reaching values of $\bar{L} \sim 3,4,5,5,6,6,7,7,8$ for $N$ $=2,4,6,8,10,12,14,16,18$, respectively. Although its actual value of $\bar{L} \sim 8$ is not large enough, one should take into account that for larger clusters, as is the case of molecules embedded in helium droplets, much higher values of the orbital angular momentum would be expected therefore leading to the correct agreement with experiments.

\section{Simulated spectra}

Considering a $(v=1 \leftarrow v=0)$ transition, application of Eq. (24) to $\mathrm{Br}_{2}(X)-\left({ }^{4} \mathrm{He}\right)_{18}$ boson complex leads to a stick vib-rotational Raman spectrum that can be compared to that for the isolated bromine in Fig. 3. To obtain the averaged spherical and anisotropic polarizabilities of $\mathrm{Br}_{2}, r$-dependent cubic polynomial fits to $a b$ initio calculations ${ }^{25}$ have been used. In this way, and assuming a rotational temperature of 2 $\mathrm{K}$, dominant $Q$ lines are found, their intensity being one order of magnitude higher than the $O$ and the $S$ lines. As our simple model predicts, the different lines corresponding to the complex appear $\approx 0.20 \mathrm{~cm}^{-1}$ shifted to the left with respect to the isolated diatomic molecule.

In order to further include broadening of the spectral lines and obtain continuum spectra for the three species mentioned above, the model described in Sec. II D 2 has been used. For pure bosons, Fig. 4 shows the angular-dependent half width for the VP process $\mathrm{Br}_{2}(X, v=1)-{ }^{4} \mathrm{He} \rightarrow \mathrm{Br}_{2}(X, v$ $=0)+{ }^{4} \mathrm{He}$ obtained by applying the model of Beswick and Delgado-Barrio. ${ }^{18}$ It predicts the presence of three maxima, at linear and perpendicular configurations, passing through zero at $\sim 60$ and $120^{\circ}$. It has to be averaged according to the polar orientation of $\mathrm{He}$ atoms with respect to the $\mathrm{Br}_{2}$ axis within the complex. Such distribution, calculated at the Hartree level, is also depicted in the figure, together with that obtained by DMC (Ref. 24) calculations. Only a qualitative agreement between the two distributions can be obtained, suggesting the presence of three rings of $\mathrm{He}$ atoms around the bromine axis. In fact, the Hartree results overestimate the $T$-shaped configurations and present a more pronounced anisotropy than the DMC ones. However, the averaged half widths, Eq. (25), are almost the same, amounting to 3.563 $\times 10^{-5} \mathrm{~cm}^{-1}$ (Hartree) and $3.117 \times 10^{-5} \mathrm{~cm}^{-1}$ (DMC), respectively. So, within the model used here, Hartree results may be considered reliable for energies and broadening of lines which determine the continuum Raman spectra.

The same quantities are depicted in Fig. 5 for the pure fermionic complex, but the angular distributions (corresponding to the two limiting spin multiplicities) were obtained through Hartree-Fock calculations. The triatomic VP half width looks similar to the bosonic case, the quantitative difference coming from the different mass of the helium atom. Also the angular distributions are qualitatively similar, the singlet state showing an even larger concentration of he-

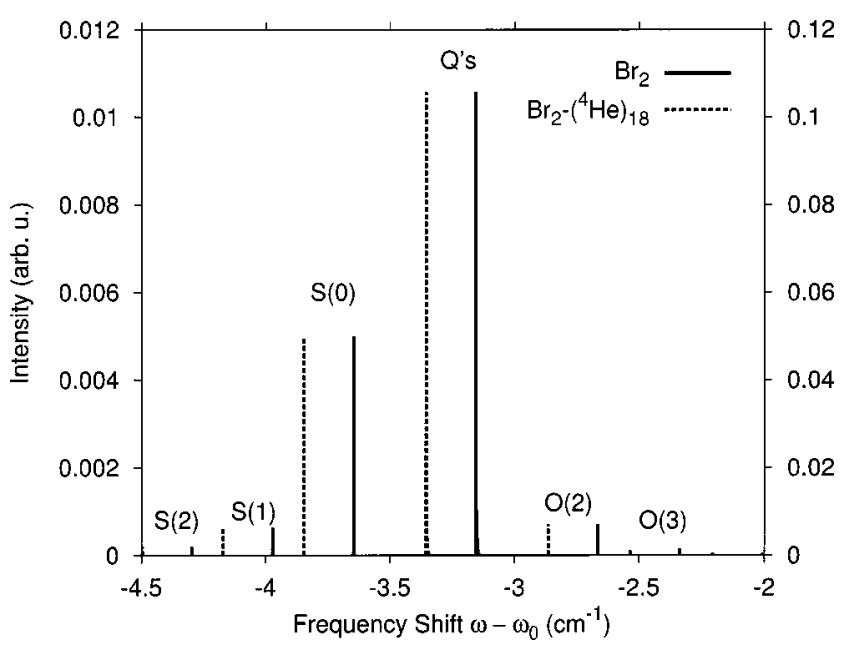

FIG. 3. Stick Raman spectra $(v=1 \leftarrow v=0)$ of $\operatorname{Br}_{2}(X)$ and $\operatorname{Br}_{2}(X)-\left({ }^{4} \mathrm{He}\right)_{18}$ species at $T=2 \mathrm{~K} . Q$ lines, presenting intensities of $\sim$ ten times greater than the other ones, are referred to the vertical axis on the right. 


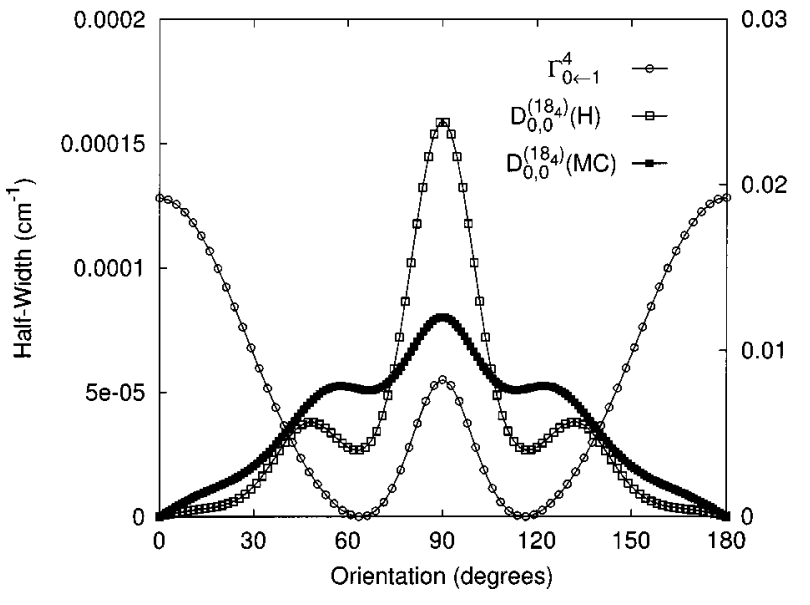

FIG. 4. Orientation dependence of the half-width for the VP process $\mathrm{Br}_{2}(X, v=1)-{ }^{4} \mathrm{He} \rightarrow \mathrm{Br}_{2}(X, v=0)+{ }^{4} \mathrm{He}$ and angular distributions for the $\mathrm{Br}_{2}(X)-\left({ }^{4} \mathrm{He}\right)_{18}$ complex obtained through DMC and Hartree calculations.

lium atoms in the $T$-shaped configuration than for bosons, while that corresponding to $S=9$ decreases such concentration and increases the population in lateral rings. Accounting for the different spin multiplicities, the averaged half widths are again almost independent of the spin, displaying an oscillating behavior that ranges from $1.004 \times 10^{-4} \mathrm{~cm}^{-1}(S$ =6) to $1.064 \times 10^{-4} \mathrm{~cm}^{-1}(S=3)$. Note that, in any case, these values are $\sim$ three times larger than in the bosonic case.

To estimate the VP half widths for the species containing 18 fermions, 18 bosons, and their addition (through Eqs. 25 and 26) the following quantities $\left(\mathrm{cm}^{-1}\right)$ have been used: $E^{1_{4}}=-17.156, \Gamma_{0 \leftarrow 1}^{4}=3.56 \times 10^{-5}, E^{\left(18_{4}\right)}=-222.17, E^{1_{3}}$ $=-14.385$, and simple averages among spin multiplicities for $\Gamma_{0 \leftarrow 1}^{3}=1.04 \times 10^{-4}, E^{\left(18_{3}\right)}=-184.84$, and $\left\langle\left\langle V_{F / B}^{18 / 18}\right\rangle\right\rangle$ $=84.83$. In this way, the half widths reached the values of $0.00133,0.00046$, and $0.00140 \mathrm{~cm}^{-1}$ for fermions, for bosons, and for the mixture, respectively.

At a rotational temperature of $2 \mathrm{~K}$, the corresponding vib-rotational Raman spectra show a dominant $Q$ branch, the $S$ (and $R$ ) branches being $\sim$ ten times less intense, and the $O$

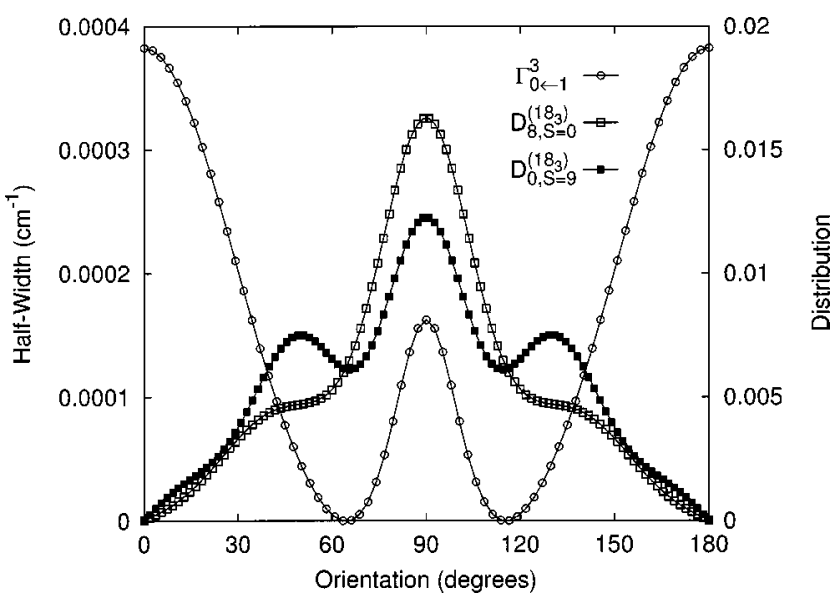

FIG. 5. Dependency on the orientation of the half-width for the VP process $\mathrm{Br}_{2}(X, v=1)-{ }^{3} \mathrm{He} \rightarrow \mathrm{Br}_{2}(X, v=0)+{ }^{3} \mathrm{He}$ and angular distributions for the $\mathrm{Br}_{2}(X)-\left({ }^{3} \mathrm{He}\right)_{18}$ complex for the two limiting spin cases, $S=0$ and $S=9$, obtained by Hartree-Fock calculations.

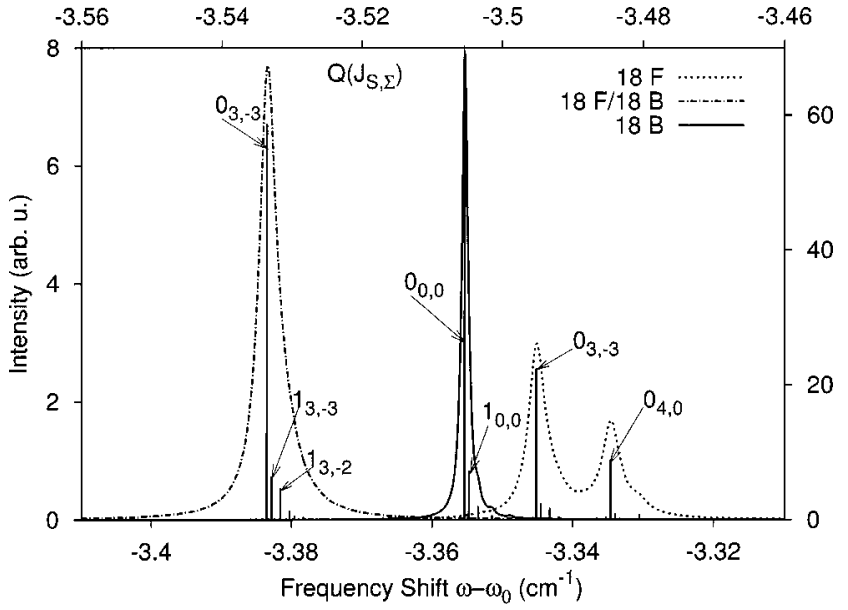

FIG. 6. $Q$ branch region showing the gradual approach from the broad profile of the complex containing pure fermions, to the apparent single peak for that corresponding to pure bosons, through the complex build up with a mixture of fermions and bosons.

(and $P$ ) branches even an order of magnitude smaller. So, we have decided to show separately these regions of the spectrum in order to perform their analysis.

For the more intense $Q$ branch, Fig. 6 shows the spectra corresponding to the three different species. The frequencies follow the sequence: fermions, bosons, and the mixing from right to left, as a manifestation of the vibrational frequency shift. For the mixture, and to make evident the correspondence, the spectrum has been shifted to the right by $0.15 \mathrm{~cm}^{-1}$, and is reported on the upper, horizontal axis. Since the bosonic spectrum is again ten times more intense than those containing fermions, it is marked on the vertical axis on the right. Using the notation $Q\left(J_{S, \Sigma}\right)$, the more important lines are included [scaled by a factor $(\pi \Gamma)^{-1}$ ]. While for pure bosons this branch is an apparent single Lorentzian profile, mainly corresponding to $J=0$, for pure fermions the presence of several contributing lines from different spin multiplicities and their projections gives rise to a much more broad and unstructured profile. In turn, and due to the loss of degeneracy, the spectrum for the fermion/boson mixture recovers the appearance of a single Lorentzian profile getting similar, albeit wider, to that shown by pure bosons. It is clearly narrower than the fermionic one.

Using a similar convention for the less intense part of the Raman spectrum, Fig. 7 displays the $O, P$ branches. Again they are more intense for pure bosons, which present only $O$ branches, and are referred to the vertical axis on the right. However, as predicted, those complexes containing fermions exhibit also $P$ branches, which sometimes are more intense than the $O$ ones [compare, e.g., $P(2)$ and $O(3)$ ]. For pure fermions, the $O$ lines are superimposed to $P$ ones $[O(2) / P(3), O(3) / P(5)$, etc.], although the former dominate over the latter. Due to the presence of $P$ branches, the effect is a congestion of the spectrum for those complexes containing fermions with respect to the pure bosons case. For complexes containing a larger number of fermionic He atoms, an amplification of such an effect may therefore be envisaged.

For the intermediate intensity, and using the conventions 


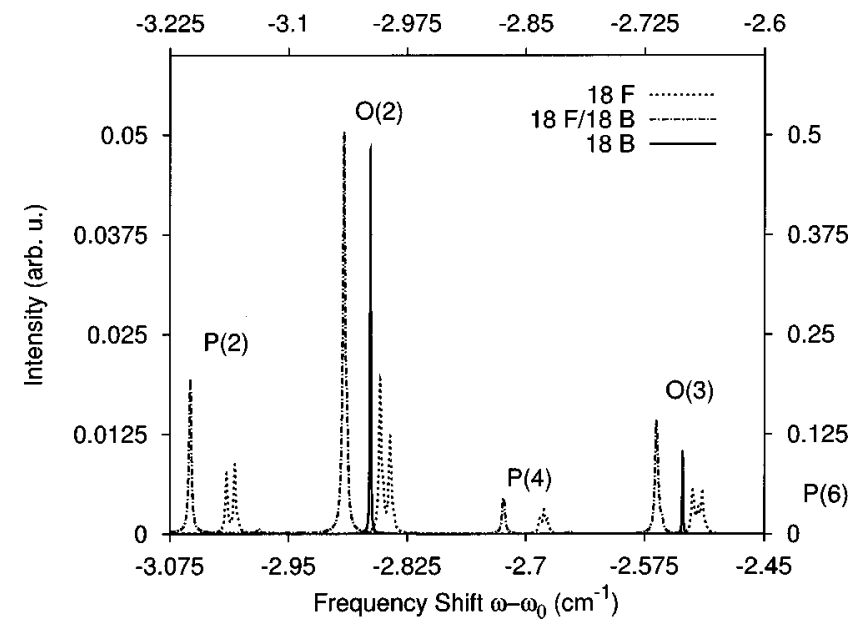

FIG. 7. $O, P$ profiles for the three species. $P$ branches are absent for complexes containing only bosons. The same conventions than in Fig. 6 have been used.

of the two previous figures, we plot in Fig. 8 the corresponding $R, S$ profiles of the Raman Spectrum. Again, only $S$ branches appear for the pure boson case, while interplaced $R$ branches do appear for complexes containing fermions, giving rise to congestion in the spectrum. For pure fermions, the $S$ branches present again some contamination from the $R$ ones $[S(0) / R(2), S(1) / R(4)$, etc.].

As an example, we display in Fig. 9 the $S(1)$ region with the assignment of the more important lines. It presents a single $S(1)$ Lorentzian profile for bosons, a rather unstructured profile for pure fermions with main contributions from $S(1)$ and also $R(4)$, and again a more simplified profile for the boson/fermion mixture coming essentially from $S(1)$ lines with negligible $R(4)$ contributions.

\section{CONCLUSIONS}

We have presented in this work quantum chemistry-type calculations for complexes of $\mathrm{Br}_{2}$ with $\mathrm{He}$ (boson or fermion) atoms. They were treated at the same level of accuracy, i.e., through Hartree and Hartree-Fock calculations, re-

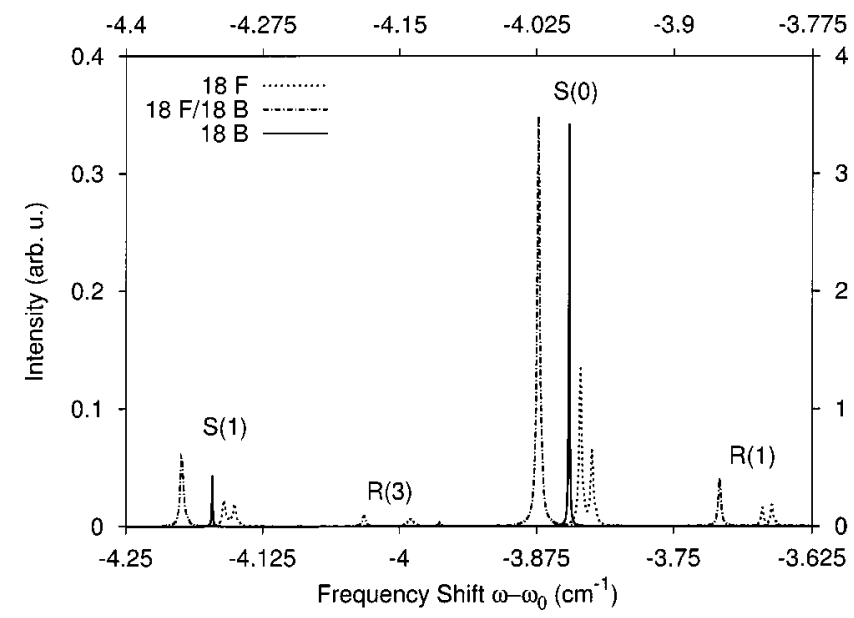

FIG. 8. $R, S$ profiles for the three species. $R$ branches are absent for complexes containing only bosons. The same conventions than in Figs. 6 and 7 have been used.

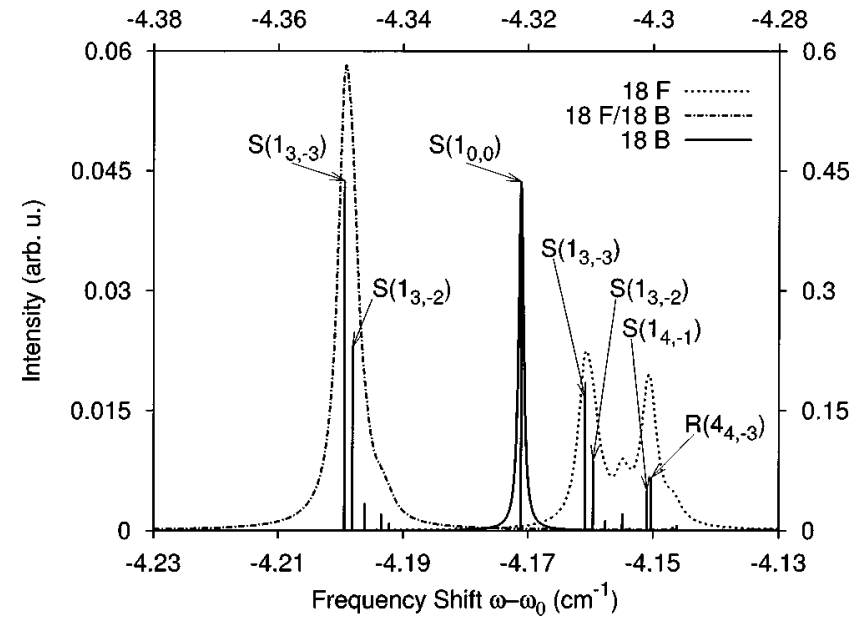

FIG. 9. Enlarged $S(1)$ region from Fig. 8.

spectively. The former has been tested against DMC calculations for sizes up to $18{ }^{4} \mathrm{He}$ atoms, using a truncated core potential for the He-He interaction. This potential depends on a single parameter which has been fitted for $N$ $=2$, and then used along all sizes, also for fermions. A simple SCF procedure has also been designed to deal with fermion/boson mixtures.

Using an adiabatic model for the intramolecular bond distance, and assuming a simple model of VP to introduce broadening effects, vib-rotational Raman spectra have been simulated for those complexes containing 18 bosons, 18 fermions, and the mixture of both.

The more intense $Q$ branch already exhibits the main characteristics experimentally found by Grebenev et al. ${ }^{1}$ for the IR spectra of OCS embedded in He droplets: (1) A simple profile for pure bosons, which apart from a little broadening and frequency shift results close to that corresponding to the isolated molecule, (2) a broad structure coming from the overlap of several lines corresponding to different spin multiplicities for the pure fermionic case, and (3) recovering of simpler structure for the boson/fermion mixture as a consequence of loss of degeneracy.

While for pure bosons only $S$ and $O$ branches are present, $R$ and $S$ interplaced structures do appear for complexes including fermions, giving rise to a congestion in the spectrum. In any case, the same behavior is also obtained for $Q$ branches for the sequence of pure bosons, mixture, and pure fermions in order of increasing complexity.

Possible extension of this work are twofold. On the one hand, we plan to study polar diatomic molecules, as $\mathrm{CO}$, HF, etc. to perform infrared simulations able to compare with the recent experiments (see e.g. Refs. 26 and 27), increasing at the same time the size of the clusters. On the other hand, we plan to use high-level Quantum Chemistry packages [as MOLPRO (Ref. 28)] through replacement of $r_{12}$ Coulomb potential terms by the appropriate ones involved in the present problem. This also suggests the possibility of dealing with different many-body interactions which appear in related fields, e.g., elementary particles or condensed matter physics. 


\section{ACKNOWLEDGMENTS}

This work has been partially supported by the ItalySpain Integrated Action Program HI02-74, the DGICYT Spanish Grant No. BFM2001-2179, and the European TMR network Grant No. HPRN-CT-1999-0005. F.A.G. and C.DiP. thank the Research Committee of the University of Rome $L a$ Sapienza. M. P. deL.-C. thanks Hendrik J. Monkhorst for useful discussions as well as the Comunidad Autonoma de Madrid (Spain) for the financial support. J.J. was supported by the Office of Basic Energy Sciences, Division of Chemical Sciences, Geosciences, and Biosciences, U.S. Department of Energy under Contract number W-31-109-Eng-38.

${ }^{1}$ S. Grebenev, J. P. Toennies, and A. F. Vilesov, Science 279, 2083 (1998).

${ }^{2}$ M. Pi, R. Mayol, and M. Barranco, Phys. Rev. Lett. 82, 3093 (1999).

${ }^{3}$ M. Barranco, M. Pi, S. M. Gatica, E. S. Hernández, and J. Navarro, Phys. Rev. B 56, 8997 (1997).

${ }^{4} \mathrm{Ph}$. Sindzingre, M. L. Klein, and D. M. Ceperley, Phys. Rev. Lett. 63, 1601 (1989).

${ }^{5}$ M. V. Rama Krishna and K. B. Whaley, Phys. Rev. Lett. 64, 1126 (1990).

${ }^{6}$ Y. Kwon, D. M. Ceperley, and K. B. Whaley, J. Chem. Phys. 104, 2341 (1996).

${ }^{7}$ V. S. Babichenko and Yu. Kagan, Phys. Rev. Lett. 83, 3458 (1999).

${ }^{8}$ P. Jungwirth and A. Krylov, J. Chem. Phys. 115, 10214 (2001).

${ }^{9}$ A. Heidenreich and J. Jortner, J. Chem. Phys. 118, 10101 (2003).

${ }^{10} \mathrm{P}$. Villarreal, O. Roncero, and G. Delgado-Barrio, J. Chem. Phys. 101, 2217 (1994).

${ }^{11}$ D. F. R. Brown, J. K. Gregory, and D. C. Clary, J. Chem. Soc., Faraday Trans. 92, 11 (1996).

${ }^{12}$ G. C. Schatz, R. B. Gerber, and M. A. Ratner, J. Chem. Phys. 88, 3709 (1988).
${ }^{13}$ J. Fernandez Rico in Direct minimization in Hartree-Fock Methods SelfConsistent Field, Theory and Applications, edited by R. Carbo and M. Klobukowski, Elsevier, Amsterdam, 70 (1990).

${ }^{14}$ E. B. Wilson, Jr., J. C. Decius, and P. C. Cross, Molecular Vibrations (Dover, New York, 1980).

${ }^{15}$ See, e.g., M. I. Hernández, T. González-Lezana, A. Buchachenko, R. Prosmiti, M. P. de Lara-Castells, G. Delgado-Barrio, and P. Villarreal, Recent Res. Devel. Chem. Physics 4, 1 (2003) and references therein.

${ }^{16}$ P. Villarreal, A. Varadé, and G. Delgado-Barrio, J. Chem. Phys. 90, 2684 (1989).

${ }^{17}$ M. I. Hernández, A. García-Vela, J. Campos-Martínez, O. Roncero, P. Villarreal, and G. Delgado-Barrio, Comput. Phys. Commun. 145, 97 (2002).

${ }^{18}$ J. A. Beswick and G. Delgado-Barrio, J. Chem. Phys. 73, 3653 (1980).

${ }^{19}$ K. P. Huber and G. Herzberg, Constants of diatomic molecules (van Nostrand Reinhold, New York, 1979).

${ }^{20}$ T. González-Lezana, M. I. Hernández, G. Delgado-Barrio, A. A. Buchachenkov, and P. Villarreal, J. Chem. Phys. 105, 7454 (1996).

${ }^{21}$ Z. Bačić, M. Kennedy-Mandziuk, J. W. Moskowitz, and K. E. Schmidt, J. Chem. Phys. 97, 6472 (1992)

${ }^{22}$ N. Bernardes and H. Primakoff, Phys. Rev. 119, 968 (1960).

${ }^{23}$ L. H. Nosanow, Phys. Rev. 146, 120 (1966); J. H. Hetherington, W. J. Mullin, and L. H. Nosanow, 154, 175 (1967)

${ }^{24}$ D. López-Duran, M. P. de Lara-Castells, G. Delgado-Barrio, P. Villarreal, C. Di Paola, F. A. Gianturco, and J. Jellinek, Chem. Phys. (to be published).

${ }^{25}$ G. Maroulis and C. Makris, Mol. Phys. 91, 333 (1997).

${ }^{26}$ J. Tang and A. R. W. McKellar, J. Chem. Phys. 119, 754 (2003).

${ }^{27}$ K. Nauta and R. E. Miller, J. Chem. Phys. 113, 10158 (2000).

${ }^{28}$ MOLPRO, a package of ab initio programs designed by H.-J. Werner and P. J. Knowles, version 2002.1., R. D. Amos, A. Bernhardsson, A. Berning, et al. Birmingham, UK, 2002. 